\title{
Corticosteroids induce chemotherapy resistance in the majority of tumour cells from bone, brain, breast, cervix, melanoma and neuroblastoma
}

\author{
CHENGWEN ZHANG ${ }^{1}$, BENJAMIN BECKERMANN ${ }^{1}$, GEORGIOS KALLIFATIDIS ${ }^{1}$, ZHENG LIU ${ }^{1}$, \\ WERNER RITTGEN ${ }^{2}$, LUTZ EDLER ${ }^{2}$, PETER BÜCHLER ${ }^{3}$, KLAUS-MICHAEL DEBATIN $^{4}$, \\ MARKUS W. BÜCHLER ${ }^{3}$, HELMUT FRIESS ${ }^{3}$ and INGRID HERR ${ }^{1,3,4}$
}

\author{
${ }^{1}$ Research Group Molecular OncoSurgery, University of Heidelberg ${ }^{2}$ Department of Biostatistics, German Cancer Research \\ Center; ${ }^{3}$ Department of General Surgery, University of Heidelberg; ${ }^{4}$ Department of Pediatrics, University of Ulm, Ulm, Germany
}

Received May 3, 2006; Accepted June 29, 2006

\begin{abstract}
Glucocorticoids (GCs) such as dexamethasone (DEX) have been widely used as co-medication in cancer therapy because they have potent proapoptotic properties in lymphoid cells, can reduce nausea, and alleviate acute toxic effects in healthy tissue. However, GCs are used in a supportive-care role, even though no prospective clinical studies have assessed the effect of these steroids on the growth of solid tumours. Data from preclinical and, to some extent, clinical studies, suggest that GCs induce treatment resistance in some solid tumours. Since it is unknown whether GC-induced resistance occurs only occasionally or is a more common phenomenon, we performed a screening study using several established cell lines from bone, brain, breast and cervix carcinoma as well as melanoma and neuroblastoma together with fresh surgical resections from patients with breast cancer. We found that DEX inhibits cisplatin and 5fluorouracil-induced apoptosis and promotes the growth of the majority of examined malignant cells. In contrast, and as expected, DEX acted pro-apoptotically and promoted the cytotoxic effect of chemotherapy in established and primary lymphoid cells. Thus, these data demonstrate the need for detailed molecular studies to clarify the mechanism of differential glucocorticoid signaling as well as controlled, prospective clinical studies.
\end{abstract}

Correspondence to: Dr Ingrid Herr, University of Heidelberg, Molecular OncoSurgery, Im Neuenheimer Feld 365, 69120 Heidelberg, Germany

E-mail: i.herr@dkfz.de

Abbreviations: DEX, dexamethasone; GCs, glucocorticoids; GR, glucocorticoid receptor; 5-FU, 5-fluorouracil

Key words: cancer therapy, glucocorticoids, nausea, apoptosis

\section{Introduction}

For nearly 50 years, physicians have relied on glucocorticoids (GCs), steroid hormones usually secreted by the body in response to stress, to treat several types of cancer. GCs can kill cancerous lymphoid cells, and are thus important for treatment of malignant hematological diseases (1). These hormones are also widely used in combination with chemotherapy and radiation of patients with solid tumours due to several other beneficial effects. Thus, GCs reduce nausea and emesis, protect healthy tissue from cytotoxic side-effects, and presumably reduce tissue reactions such as inflammation against invasive malignant growth $(2,3)$.

Due to these multiple benefits, GCs are widely used as co-medication in cancer therapy of lymphoid and solid tumours and several leading clinical oncology organizations have endorsed the use of GCs in a supportive-care role. While GCs generally support therapy of lymphoid tumour cells, some studies describe inhibition of cancer therapy in some cell lines of solid tumours (reviewed in ref. 4). Concomitantly, concerns about the widespread use of GCs during therapy of solid tumours have been expressed repeatedly $(5,6)$ involving studies showing enhanced percentages of metastases in breast cancer patients $(7,8)$ or an increased risk of skin cancer and non-Hodgkin lymphomas among users of systemic GCs (9). Remarkably, prospective randomized clinical trials evaluating any potential impact of GCs on tumour control have never been performed. Most importantly, it is not even known, whether GC-conferred resistance in solid tumours is a common or only an occasional problem.

We present a screening study of DEX-induced resistance in established and primary tumour cells from bone, brain, breast, cervix, melanoma and neuroblastoma. In the presence of cisplatin or 5-fluorouracil (5-FU), DEX substantially increased viability or inhibited apoptosis of most examined tumour cells of solid, but not of lymphoid, malignant diseases. Resistance induced by DEX was evaluated by a new statistical method, which has been exclusively designed by us for the definition of resistance. Using this method, we found significant resistance in 23 of 26 examined established carcinoma cell lines and in 
1 of 2 examined freshly resected breast tumour specimens. These data on analysed primary and established cell lines represent an important reference database for those interested in the pharmacology of the GC/chemotherapy interaction and demonstrate the need for mechanistic and controlled clinical prospective studies.

\section{Materials and methods}

Established cell lines. Cell lines of the following origin of tumours or tumour types were used: bone: MG63, HOS, SAOS, HT1080; brain: HS683, H4, A172 (glioma), U-373MG (glioblastoma/astrocytoma), TE671 (medulloblastoma); breast: BT-474, BT-20, Colo-824, MCF7, MDAMB-436; cervix: P5, CASKI, MRH-215, MRH-186, MRH-196; melanoma: HS695T, WM1341, WM98.1; neuroblastoma: IMR32, KELLY, SKN-SH, SHEP, IMR5; T cell leukemia: CEM. The cell lines were cultured as described in our recent studies (10-15).

Isolation of fresh breast tumour cells and TALs. Breast tumours were resected, inspected by a pathologist, and the selected pieces containing at least $80 \%$ tumour cells were transported in Liforlab medium (Oncoscience, Wedel, Germany) to the laboratory. The fresh tissues were minced in RPMI medium supplemented with $20 \%$ heat-inactivated fetal bovine serum (Sigma, Deisenhofen, Germany), 25 mM HEPES, 2 mM Lglutamine and Pen/Strep (all from Gibco/Life Technologies, Paisley, UK) under sterile conditions, counted by trypan blue exclusion and immediately analyzed by the MTT-assay. TALs (tumour-associated lymphocytes) were derived from ascites of a patient with ovarian carcinoma, in the ascites there were tumour cells together with lymphocytes which were separated by Ficoll gradient centrifugation. Patient material was obtained under the approval of the ethics committee of the University of Heidelberg. Diagnoses were established by conventional clinical and histological criteria according to the World Health Organization (WHO). All surgical resections were indicated by principles and practice of oncological therapy.

Cytotoxic treatment. Stock solutions of cisplatin and 5-FU (Sigma) were prepared in DMSO. Gemcitabine (kindly provided by MSD, USA) was diluted in cell culture medium. Stock solutions of DEX (Sigma) were prepared in ethanol. Final concentrations of the solvents in medium were $0.01 \%$ or less.

Measurement of apoptosis. Cells were stained with fluoresceinthiocyanate (FITC)-conjugated annexin V (BD Biosciences, Heidelberg, Germany) and externalization of phosphatidylserine as well as the forward side scatter profile were identified by flow cytometry (FACScan, BD Biosciences) as described (10).

MTT-assay. Tumour cells were resuspended at $5 \times 10^{4}-10^{5} / \mathrm{ml}$ in 96-well microplates, $100 \mu 1$ per well. In detail, we used a concentration of $5 \times 10^{5} / \mathrm{ml}$ for all established cell lines and most freshly isolated tumour cells except those from which only minimal amounts of tissue were available. However, using less than $5 \times 10^{5}$ cells $/ \mathrm{ml}$ did not change the results but only prolonged the incubation time with the MTT-agent. After treatment, the MTT-assay was performed as described (16).

\section{Statistical analysis}

Surgical specimens. Each tumour probe was investigated in a 2-factorial design consisting of three doses of DEX $(0.1 \mu \mathrm{M}$ $=\mathrm{DEX} 1,1 \mu \mathrm{M}=\mathrm{DEX} 2,10 \mu \mathrm{M}=\mathrm{DEX} 3)$ and a control and three doses of cytotoxic treatment and a control $(=0)$ resulting in a total of 16 experimental conditions. Viability of the cells under each condition was determined as the mean of 3 to 8 replicates together with its standard deviation and then standardized on the result of the double control (no cytotoxic agent and no DEX applied); i.e. the viabilities of all conditions were divided by the mean of the double control. For each sample the standardized means were compared separately for each therapeutic dose and for the control by comparing the three DEX doses with its respective control (cytotoxic treatment alone). The four means (three DEX doses and control) under the condition of no therapy describe the effect of DEX alone while the other three sets of four means under the three doses of the therapeutic agent describe the resistance of the cells under treatment depending on DEX. We declared a DEX dose group as significant resistant when its mean minus one standard deviation $\left(\bar{X}_{j}-S D_{j}\right)$ was still larger than the mean plus one standard deviation $\left(\bar{X}_{0}+S D_{0}\right)$ of the respective control group of that tumour sample, $\mathrm{j}=1,2,3$ denoting the three dose groups receiving cytotoxic drugs. On this basis, scores were calculated per therapeutic dose (values $0,1,2,3$ ) as well as per surgical specimen in total (values ranging from 0 to 9 ). A tumour probe was declared as being significantly resistant to cytotoxic treatment when it showed a score of 2 for at least one drug dose or when it reached the total score of 5 out of a maximum of 9 per time point (i.e. $>50 \%$ ). The scores and definition of resistance have been exclusively designed for the present study by the Department of Biostatistics, German Cancer Research Center.

Established cell lines. Corresponding to the analysis of patientderived tumour probes, cell lines were investigated in a 2factorial design consisting of one dose of DEX $(1 \mu \mathrm{M})$ and a control and three doses of cytotoxic treatment and a control resulting in a total of 8 experimental conditions. Resistance of the cells under each condition was determined as mean of 8 replicates (MTT-assay) or as mean of 6 replicates (apoptosis) as described above.

\section{Results}

DEX induces resistance towards chemotherapy in breast cancer cells. To evaluate whether DEX could protect breast cancer cells we freshly isolated tumour cells from surgical specimens of breast tissue from 2 patients. The histology of the tissue was inspected by a pathologist. DEX was pre-incubated in concentrations of $0.1,1$ and $10 \mu \mathrm{M}$ from which the median concentration resembles peak plasma levels found in the clinical setting (17-19). Cells were treated with three different concentrations of cisplatin $24 \mathrm{~h}$ after pre-incubation. After an additional $48 \mathrm{~h}$, viability was measured and the MTT-data were normalized to untreated control cells. The statistical significance of DEX-induced resistance was analyzed (Table I, 
A
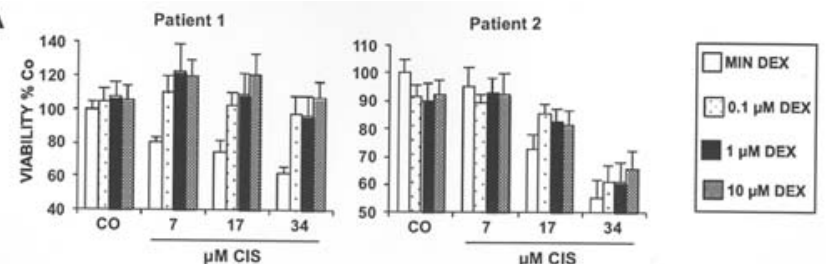

B

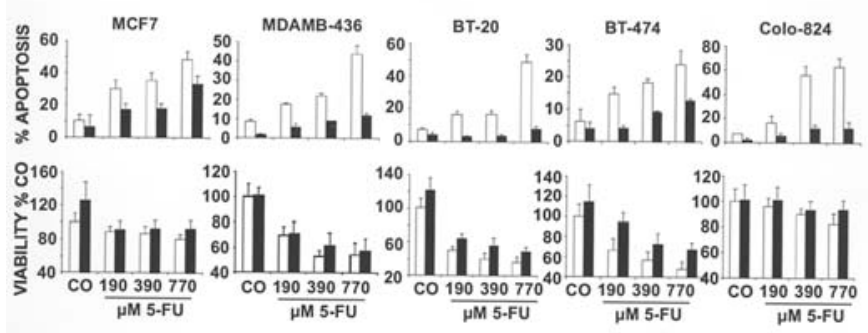

C

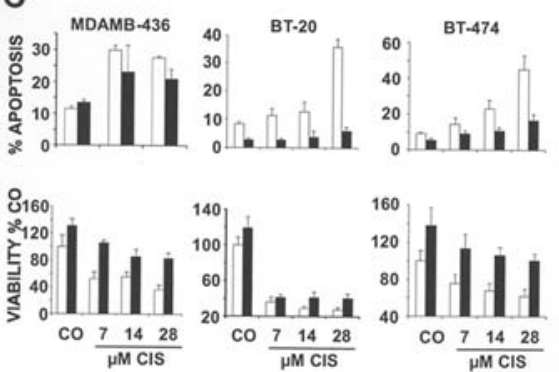

Figure 1. DEX inhibits apoptosis and promotes proliferation in breast cancer cells. (A) Tumour cells from surgical resections from two patients with breast cancer were freshly isolated and cultivated in a concentration of $5 \times 10^{5} / \mathrm{ml}$ in the absence (white bars) or presence of DEX $(0.1,1$ or $10 \mu \mathrm{M}$ as indicated) for $24 \mathrm{~h}$. Cisplatin (CIS) was added $(7,17$ or $34 \mu \mathrm{M}$ as indicated) while the controls remained either untreated $(\mathrm{CO})$ or were treated with DEX alone. Viability was measured by the MTT-assay $48 \mathrm{~h}$ after adding cisplatin. Eight wells per treatment were analyzed and standard deviations are $<10 \%$. Statistical analysis of significance of DEX-induced resistance is shown in Table $\mathrm{I}$. (B) The established breast cancer cells MCF7, MDAMB-436, BT-20, BT-474 and Colo-824 were left either untreated (CO) or were treated with (B) 5 -FU $(190,390$ or $770 \mu \mathrm{M})$ or $(\mathrm{C})$ cisplatin (CIS: 7, 14, $28 \mu \mathrm{M}$ ) in the absence (white bars) or presence (black bars) of DEX $(1 \mu \mathrm{M})$ which was added $48 \mathrm{~h}$ prior to cytotoxic treatment. Apoptosis was analyzed by staining the cells with annexin-FITC and FACSanalysis $72 \mathrm{~h}$ after the addition of drugs. Likewise, viability was detected by the MTT-assay. Experiments were performed three times with identical outcome and standard deviations are shown. Statistical analysis of significance of resistance is shown in Table II.

Fig. 1A). While cytotoxic treatment strongly reduced viability, the presence of DEX enhanced viability significantly in patient 1. Enhanced viability was also found in patient 2 at higher cisplatin concentrations although the observed differences were not significant.

To further investigate DEX-induced resistance in breast cancer, we treated MCF7, MDAMB-436, BT-20, BT-474 and Colo-824 cells with 5-FU (Fig. 1B) or cisplatin (Fig. 1C) in the presence or absence of DEX which was pre-incubated prior to the addition of drugs. Apoptosis was detected by annexin-FITC $72 \mathrm{~h}$ after cytotoxic treatment followed by FACS-analysis. While cytotoxic drugs alone strongly induced apoptosis, the presence of DEX inhibited this effect in all cell lines examined. No induction of apoptosis occurred in untreated cells or cells treated with DEX alone. Considering the inhibited apoptosis we next analyzed whether DEX might influence the growth of breast carcinoma cell lines treated
Table I. DEX-induced resistance in primary cells from resected breast tumour specimens.

\begin{tabular}{cccc}
\hline Tissue & Patient probe & Drug & Significance \\
\hline Breast & $1,+$ & CIS &,$+ \mathrm{s}$ \\
& $2,-$ & CIS &,$+ \mathrm{ns}$
\end{tabular}

+, resistance; -, no resistance; s, significant; ns, not significant (for definition of significance see Materials and methods). ${ }^{\text {aNumber of }}$ tumours in which significant DEX-mediated enhanced viability was

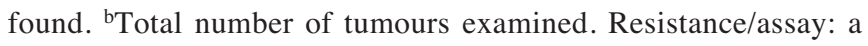
tumour probe per assay was defined to be resistant when at least $50 \%$ of cells showed enhanced viability in response to $0.1,1$ and $10 \mu \mathrm{M}$ DEX compared to the respective control cells, this definition depends on the median numbers and is irrelevant of significance; a tumour was defined to be non-resistant when at least $50 \%$ of cells showed reduced or similar viability in response to $0.1,1$ and $10 \mu \mathrm{M}$ DEX compared to the respective control cells, this definition depends on the median numbers and is irrelevant of significance. Resistance/ patient probe: depending on the results of several single assays performed per tumour, a particular tumour was defined to be resistant when the majority of the assays indicated resistance and at least one of the assays showed significant resistance.
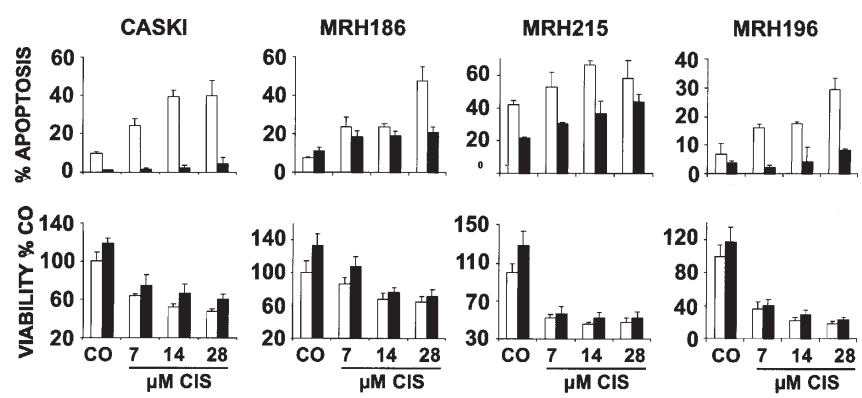

Figure 2. DEX inhibits apoptosis and promotes proliferation in cervical cancer cells. CASKI, MRH186, MRH215 and MRH196 cervix carcinoma cell lines were pre-treated with DEX followed by the addition of cytotoxic drugs in the concentrations indicated. Cells and DEX-induced resistance were analyzed as described in Fig. 1.

with cytotoxic drugs. The same set of cells was treated as described above and viability was detected by the MTT-assay. While 5-FU or cisplatin alone strongly reduced viability, the presence of DEX diminished the cytotoxic effect in all cell lines. Moreover, DEX alone enhanced basal viability and even lowered basal apoptosis in most cases. Significant induction of resistance was found in all five examined breast cancer cell lines (Table II).

DEX induces resistance towards chemotherapy in cervical cancer cells. To investigate whether DEX might interfere with chemotherapy of cervical carcinomas, CASKI, MRH186, MRH215 and MRH196 cells were treated with cisplatin (Fig. 2) in the presence or absence of pre-incubated DEX. After $72 \mathrm{~h}$, exposure of phosphatidylserine was analysed by flow cytometry and viability by the MTT-assay. While drugs alone strongly induced apoptosis and reduced viability, the presence of DEX inhibited these effects with statistical 
Table II. Significance of DEX-induced resistance in established carcinoma cell lines.

\begin{tabular}{ccccc}
\hline Tissue & Cell line & Assay & Drug & Significance \\
\hline Brain & A172, + & apoptosis & CIS &,$+ \mathrm{ns}$ \\
& MTT & CIS &,$+ \mathrm{s}$ \\
& $\mathrm{H} 4,+$ & apoptosis & CIS &,$+ \mathrm{s}$ \\
& MTT & CIS &,$+ \mathrm{s}$ \\
& HS683, + & apoptosis & CIS &,$+ \mathrm{s}$ \\
& MTT & CIS &,$+ \mathrm{ns}$ \\
& U-373-MG,+ & apoptosis & CIS &,$+ \mathrm{s}$ \\
& MTT & CIS &,$+ \mathrm{ns}$ \\
& TE671, + & apoptosis & CIS &,$+ \mathrm{s}$ \\
& MTT & CIS &,$+ \mathrm{ns}$
\end{tabular}

Breast

\begin{tabular}{|c|c|c|c|}
\hline MCF7, + & $\begin{array}{c}\text { apoptosis } \\
\text { MTT }\end{array}$ & $\begin{array}{l}5-\mathrm{FU} \\
5-\mathrm{FU}\end{array}$ & $\begin{array}{c}+, \mathrm{s} \\
+, \mathrm{ns}\end{array}$ \\
\hline MDAMB-436, + & $\begin{array}{c}\text { apoptosis } \\
\text { MTT } \\
\text { apoptosis } \\
\text { MTT }\end{array}$ & $\begin{array}{l}5-\mathrm{FU} \\
5-\mathrm{FU} \\
\text { CIS } \\
\text { CIS }\end{array}$ & $\begin{array}{c}+, \mathrm{s} \\
+, \mathrm{ns} \\
+, \mathrm{s} \\
+, \mathrm{s}\end{array}$ \\
\hline BT-20, + & $\begin{array}{l}\text { apoptosis } \\
\text { MTT } \\
\text { apoptosis } \\
\text { MTT }\end{array}$ & $\begin{array}{l}5-\mathrm{FU} \\
5-\mathrm{FU} \\
\text { CIS } \\
\text { CIS }\end{array}$ & $\begin{array}{c}+, \mathrm{s} \\
+, \mathrm{s} \\
+, \mathrm{s} \\
+, \mathrm{ns}\end{array}$ \\
\hline BT474, + & $\begin{array}{l}\text { apoptosis } \\
\text { MTT } \\
\text { apoptosis } \\
\text { MTT }\end{array}$ & $\begin{array}{l}5-\mathrm{FU} \\
5-\mathrm{FU} \\
\text { CIS } \\
\text { CIS }\end{array}$ & $\begin{array}{l}+, \mathrm{s} \\
+, \mathrm{s} \\
+, \mathrm{S} \\
+, \mathrm{s}\end{array}$ \\
\hline Colo-824, + & $\begin{array}{c}\text { apoptosis } \\
\text { MTT }\end{array}$ & $\begin{array}{l}5-\mathrm{FU} \\
5-\mathrm{FU}\end{array}$ & $\begin{array}{c}+, \mathrm{s} \\
+, \mathrm{ns}\end{array}$ \\
\hline CASKI, + & $\begin{array}{c}\text { apoptosis } \\
\text { MTT }\end{array}$ & $\begin{array}{l}\text { CIS } \\
\text { CIS }\end{array}$ & $\begin{array}{l}+, \mathrm{S} \\
+, \mathrm{S}\end{array}$ \\
\hline MRH186, + & $\begin{array}{c}\text { apoptosis } \\
\text { MTT }\end{array}$ & $\begin{array}{l}\text { CIS } \\
\text { CIS }\end{array}$ & $\begin{array}{l}+, \mathrm{S} \\
+, \mathrm{S}\end{array}$ \\
\hline MRH215, + & $\begin{array}{c}\text { apoptosis } \\
\text { MTT }\end{array}$ & $\begin{array}{l}\text { CIS } \\
\text { CIS }\end{array}$ & $\begin{array}{c}+, \mathrm{s} \\
+, \mathrm{ns}\end{array}$ \\
\hline MRH196, + & apoptosis & CIS &,$+ \mathrm{s}$ \\
\hline WT1341, + & $\begin{array}{c}\text { apoptosis } \\
\text { MTT }\end{array}$ & $\begin{array}{l}\text { CIS } \\
\text { CIS }\end{array}$ & $\begin{array}{l}+, \mathrm{s} \\
+, \mathrm{ns}\end{array}$ \\
\hline WM.98.1, + & $\begin{array}{c}\text { apoptosis } \\
\text { MTT }\end{array}$ & $\begin{array}{l}\text { CIS } \\
\text { CIS }\end{array}$ & $\begin{array}{l}+, \mathrm{s} \\
+, \mathrm{ns}\end{array}$ \\
\hline HS695T, - & $\begin{array}{c}\text { apoptosis } \\
\text { MTT }\end{array}$ & $\begin{array}{l}\text { CIS } \\
\text { CIS }\end{array}$ & $\begin{array}{l}+\mathrm{ns} \\
+, \mathrm{ns}\end{array}$ \\
\hline IMR5, - & $\begin{array}{c}\text { apoptosis } \\
\text { MTT }\end{array}$ & $\begin{array}{l}\text { CIS } \\
\text { CIS }\end{array}$ & $\begin{array}{l}+, \mathrm{ns} \\
+, \mathrm{ns}\end{array}$ \\
\hline KELLY, + & $\begin{array}{l}\text { apoptosis } \\
\text { MTT } \\
\text { apoptosis } \\
\text { MTT }\end{array}$ & $\begin{array}{l}\text { CIS } \\
\text { CIS } \\
5-F U \\
5-F U\end{array}$ & $\begin{array}{l}+, \mathrm{s} \\
+, \mathrm{s} \\
+, \mathrm{s} \\
+, \mathrm{ns}\end{array}$ \\
\hline SKNSH, + & $\begin{array}{c}\text { apoptosis } \\
\text { MTT }\end{array}$ & $\begin{array}{l}\text { CIS } \\
\text { CIS }\end{array}$ & $\begin{array}{l}+, \mathrm{s} \\
+, \mathrm{ns}\end{array}$ \\
\hline SHEP, + & $\begin{array}{l}\text { apoptosis } \\
\text { MTT } \\
\text { apoptosis } \\
\text { MTT }\end{array}$ & $\begin{array}{c}\text { CIS } \\
\text { CIS } \\
5-F U \\
5-F U\end{array}$ & $\begin{array}{l}+, \mathrm{s} \\
+, \mathrm{s} \\
+, \mathrm{s} \\
+, \mathrm{s}\end{array}$ \\
\hline
\end{tabular}

Table II. Continued.

\begin{tabular}{lcccc}
\hline Tissue & Cell line & Assay & Drug & Significance \\
\hline & IMR32, - & apoptosis & CIS & - \\
MTT & CIS & - \\
& & apoptosis & 5-FU &,$+ \mathrm{ns}$ \\
MTT & $5-\mathrm{FU}$ &,$+ \mathrm{ns}$ \\
Osteosarcoma & SaOs, + & apoptosis & CIS &,$+ \mathrm{s}$ \\
& & MTT & CIS &,$+ \mathrm{s}$ \\
& MG63, + & apoptosis & CIS &,$+ \mathrm{s}$ \\
& & MTT & CIS &,$+ \mathrm{s}$ \\
& HT1080, + & apoptosis & CIS &,$+ \mathrm{s}$ \\
& & MTT & CIS &,$+ \mathrm{s}$ \\
& HOS,+ & apoptosis & CIS &,$+ \mathrm{s}$ \\
& & MTT & CIS &,$+ \mathrm{ns}$ \\
\hline
\end{tabular}

+ , resistance; -, no resistance; s, significant; ns, not significant (for definition of significance see Materials and methods); ARA-C, cytarabine; CIS, cisplatin; GEM, gemcitabine; 5-FU, 5-fluorouracil; TAX, paclitaxel; VP-16, etoposide; Gamma, $\gamma$-irradiation. ${ }^{\text {aNumber }}$ of cell lines in which significant DEX-mediated enhanced viability was found; btotal number of cell lines examined. Resistance/assay: a cell line per assay and treatment was defined to be resistant when at least $50 \%$ of cells showed enhanced viability in response to $1 \mu \mathrm{M}$ DEX compared to the respective control cells, this definition depends on the median numbers and is irrelevant of significance; a cell line per assay and treatment was defined to be non-resistant when $>50 \%$ of cells showed reduced or equal viability in response to $1 \mu \mathrm{M}$ DEX compared to the respective control cells, this definition depends on the median numbers and is irrelevant of significance. Resistance/cell line: depending on the results of several single assays performed per cell line, a particular cell line was defined to be resistant when the majority of the assays indicated resistance and at least one of the assays showed significant resistance.

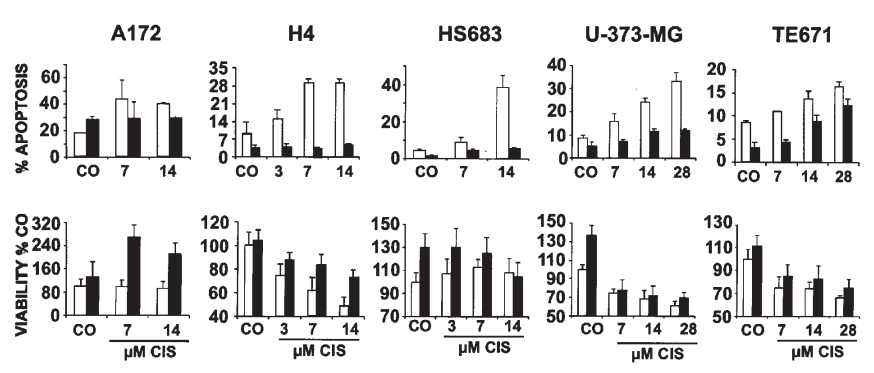

Figure 3. DEX inhibits apoptosis and promotes proliferation in brain tumour cells. A172, H4, HS683, U-373-MG AND TE671 brain tumour cell lines were pre-treated with DEX followed by the addition of cytotoxic drugs in the concentrations indicated. Cells and DEX-induced resistance were analyzed as described in Fig. 1.

significance under all conditions (Table II). Moreover, DEX alone enhanced basal viability and even lowered basal apoptosis in most cases.

DEX induces resistance towards chemotherapy in brain tumour cells. To investigate whether DEX might interfere with the chemotherapy of brain tumour cells, A172, H4, HS683, U-373- 


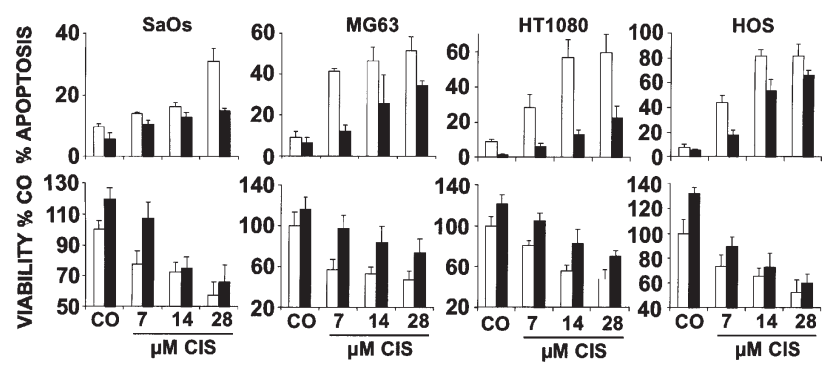

Figure 4. DEX inhibits apoptosis and promotes proliferation in bone tumour cells. SaOs, MG63, HOS (osteosarcoma) and HT1080 (fibrosarcoma) bone tumour cell lines were pre-treated with DEX followed by the addition of cytotoxic drugs in the concentrations indicated. Cells and DEX-induced resistance were analyzed as described in Fig. 1.

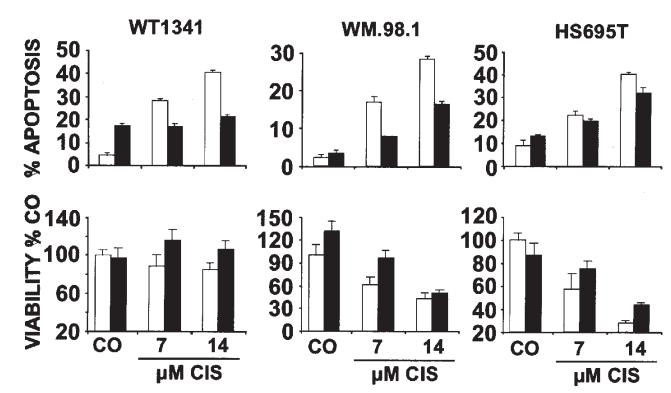

Figure 5. DEX inhibits apoptosis and promotes proliferation in melanoma cells. WT1341, WM.98.1 and HS695T melanoma cell lines were pre-treated with DEX followed by the addition of cytotoxic drugs in the concentrations indicated. Cells and DEX-induced resistance were analyzed as described in Fig. 1.

MG and TE671 cells were treated with cisplatin (Fig. 3) in the presence or absence of pre-incubated DEX. After $72 \mathrm{~h}$, the exposure of phosphatidylserine was analysed by flow cytometry and viability by the MTT-assay. While drugs alone strongly induced apoptosis and reduced viability, the presence of DEX inhibited these effects with statistical significance under all conditions (Table II). Moreover, DEX alone enhanced basal viability and even lowered basal apoptosis in most cases.

DEX induces resistance towards chemotherapy in osteosarcoma cells. To investigate whether DEX might interfere with the chemotherapy of osteosarcoma cells, SaOs, MG63, HT1080 and HOS cells were treated with cisplatin (Fig. 4) in the presence or absence of pre-incubated DEX. After $72 \mathrm{~h}$, exposure of phosphatidylserine was analysed by flow cytometry and viability by the MTT-assay. While drugs alone strongly induced apoptosis and reduced viability, the presence of DEX inhibited these effects with statistical significance under all conditions (Table II). Moreover, DEX alone enhanced basal viability and even lowered basal apoptosis in most cases.

DEX induces resistance towards chemotherapy in melanoma cells. To investigate whether DEX might interfere with the chemotherapy of osteosarcoma cells, WT1314, WM.98.1 and HS695T cells were treated with cisplatin (Fig. 5) in the presence or absence of pre-incubated DEX. After $72 \mathrm{~h}$, exposure of phosphatidylserine was analysed by flow cytometry and viability by the MTT-assay. While drugs alone
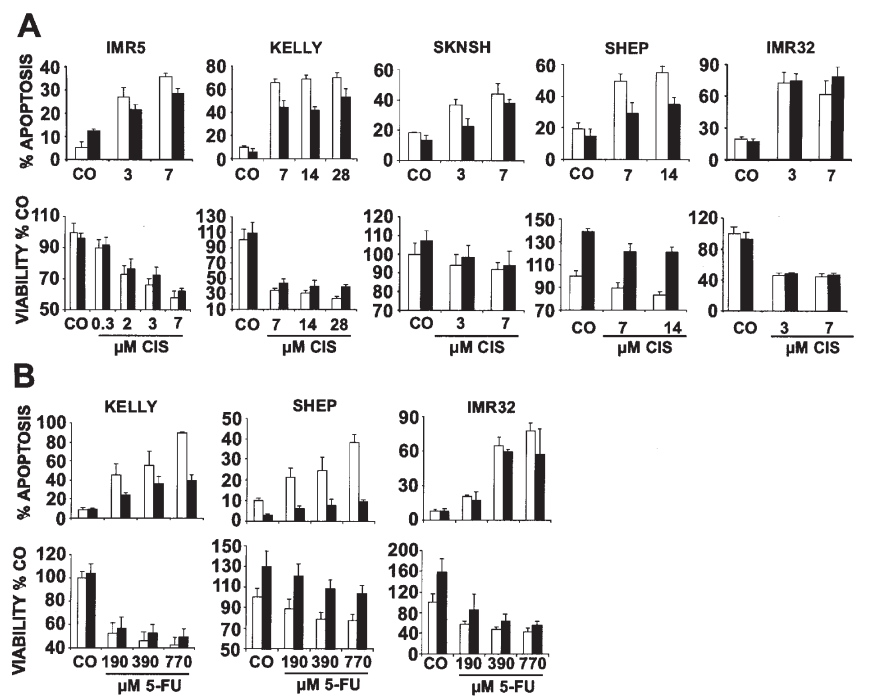

Figure 6. DEX inhibits apoptosis and promotes proliferation in neuroblastoma cells. IMR5, KELLY, SKNSH, SHEP and IMR32 cell lines were pre-treated with DEX followed by the addition of cytotoxic drugs in the concentrations indicated. Cells and DEX-induced resistance were analyzed as described in Fig. 1

A

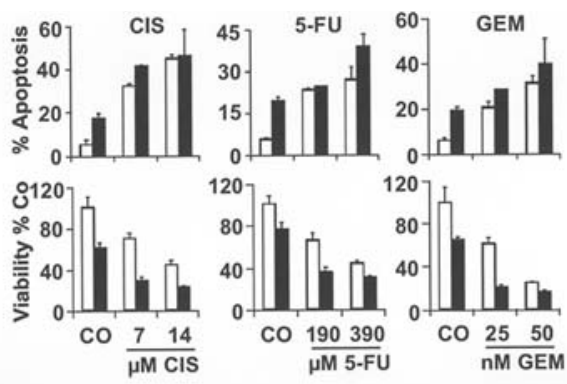

B

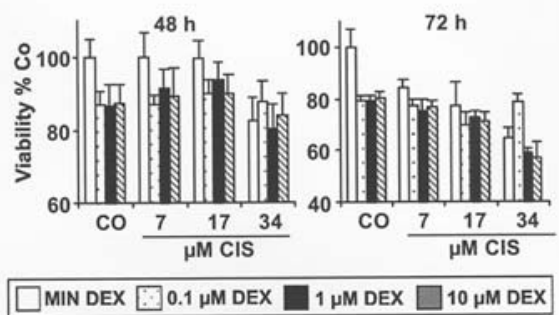

Figure 7. DEX promotes apoptosis and inhibits proliferation in lymphoid cells. (A) CEM leukemic T cells were left either untreated (CO) or were treated with cisplatin (CIS), 5-fluorouracil (5-FU) or gemcitabine (GEM) at the concentrations indicated in the absence (white bars) or presence (black bars) of DEX $(1 \mu \mathrm{M})$. Apoptosis was analyzed by annexin-staining and FACS-analysis and viability by the MTT-assay $48 \mathrm{~h}$ after cytotoxic treatment. (B) Tumor associated lymphocytes (TALs) were isolated from ascites of a patient with ovarian carcinoma. TALs were treated and analyzed as described above. Mean values \pm s.d. are shown.

strongly induced apoptosis and reduced viability, the presence of DEX inhibited these effects with statistical significance in WT13 and WM.98.1 cells (Table II). DEX-reduced apoptosis and enhanced viability was also observed in HS695T cells, although it was not statistically significant. Also, and in contrast to the other carcinoma cells examined, DEX alone enhanced basal viability only in WM.98.1 cells and did not lower basal apoptosis in any case. 
DEX induces resistance towards chemotherapy in neuroblastoma cells. To investigate whether DEX might interfere with the chemotherapy of neuroblastoma cells, IMR5, KELLY, SKNSH, SHEP and IMR32 cells were treated with cisplatin or 5-FU (Fig. 6) in the presence or absence of pre-incubated DEX. After $72 \mathrm{~h}$, the exposure of phosphatidylserine was analysed by flow cytometry and viability by the MTT-assay. While drugs alone strongly induced apoptosis and reduced viability, the presence of DEX inhibited these effects with statistical significance in KELLY, SKNSH and SHEP cells while the protective effect found in IMR5 and IMR32 cells was not significant (Table II). However, DEX alone enhanced basal viability and even lowered basal apoptosis in most cases.

DEX acts pro-apoptotically in lymphoid cells. In contrast to the data obtained with carcinoma cells and as expected, DEX did not induce resistance in lymphoid cells but rather sensitized them to therapy as shown for the leukemic T cell line CEM (Fig. 7A) and freshly isolated tumor-associated lymphocytes (TALs) from ascites of patients with ovarian cancer (Fig. 7B). Thus, the present data suggest that GC-induced resistance toward cytotoxic therapy is not an exception but common to several types of solid tumours. Together, we found significant resistance in 1 of 2 examined fresh breast cancer specimens (Table I) and in 23 of 26 examined established cell lines (Table II) from brain $(5 / 5)$, breast (5/5), cervical (4/4) and bone (4/4) carcinomas as well as from melanomas $(2 / 3)$ and neuroblastomas $(3 / 5)$.

\section{Discussion}

We report here, that DEX inhibits drug-induced apoptosis and promotes proliferation in several established and primary carcinoma cancer cells while the opposite effect occurs in lymphoid cells. These results suggest a cell type specific effect of GCs, since these agents are well known to be proapoptotic and anti-proliferative in mononuclear cells, especially lymphocytes (20) such as B cells and T cells, which are crucial for rejecting tumour cells. Increased cellular apoptosis is a main reason for the use of GCs in the treatment of many lymphoid diseases including lymphocytic cancers. However, our data demonstrate induction of therapy resistance by GCs in cells of several solid tumours along with induction of apoptosis in lymphoid cells. Recent reports identified GC-induced resistance also in other cells of solid tumours when used with various anticancer drugs and with radiotherapy. Such observations were made in established carcinoma cell lines cultured in vitro, in xenografts on nude mice, and in primary cells that had been isolated from fresh surgical samples of solid tumours (reviewed in ref. 4). In the presence of various anticancer agents, several GC derivatives, in various doses, such as betamethasone, dexamethasone, corticosterone, fluocinolone, hydrocortisone, methylprednisolone, prednisolone, and prednisone substantially increased viability or inhibited apoptosis, or both, of most tumour cells of solid, but not of lymphoid, malignant diseases (Herr I, unpublished data). GCs blocked the lethal signal delivered by cytotoxic drugs even if they were given several hours after induction of apoptosis by cytotoxic drugs. Moreover, resistance induced by a single treatment of established tumour cells with GCs lasted for up to 10 days before it started to decline, and resistance was noted at peak plasma concentrations and below (Herr I, unpublished data). Because low concentrations occur in patients after plasma concentrations of administered GCs decline, the issue of whether normal concentrations of GCs in the body could interfere with cancer treatment is raised. This point is answered by a retrospective study (21) showing an association between increased serum cortisol concentrations and high tumour grade in 211 consecutive patients with renal-cell carcinoma before starting treatment. Concentration of serum cortisol was significantly higher in patients with renal-cell carcinoma than in those with benign cysts. Serum cortisol concentration was associated with tumour diameter and grade, but not with disease stage. The outlook of patients with raised serum cortisol concentrations was worse than that of those with low concentrations. These findings with endogenous cortisol concentrations lend support to the in vitro and animal studies that showed co-treatment with GCs induced resistance to cancer. Furthermore, such findings necessitate assessment of the hypothalamic-pituitary adrenal axis in patients with malignant disease, and its possible effect on outlook.

GCs suppress the immune system, and many studies have shown that immunosuppression can exacerbate the metastatic process and accelerate tumour growth in animals (22-24). Thus, downregulation of the immune system's attack on the tumour along with induction of resistance in solid tumour cells might contribute to the observed increased metastatic potential in patients with breast cancer who receive combination treatment with GCs and the increased risk of skin cancer and lymphoma in users of systemic GCs (7-9, reviewed in ref. 4).

In conclusion, our present data together with in vitro animal and several clinical studies suggest that GCs induce resistance of carcinomas to chemotherapy and radiotherapy. However, any conclusions drawn from these insufficiently controlled retrospective clinical trials and laboratory studies need to be confirmed by well-designed clinical trials.

\section{Acknowledgements}

We thank M. Mildenberger for excellent technical assistance and the Tumorzentrum Heidelberg for financial support.

\section{References}

1. Planey SL and Litwack G: Glucocorticoid-induced apoptosis in lymphocytes. Biochem Biophys Res Commun 279: 307-312, 2000 .

2. Aapro MS: Present role of corticosteroids as antiemetics. Springer Verlag, Berlin, 1991.

3. Kirkbride P, Bezjak A, Pater J, et al: Dexamethasone for the prophylaxis of radiation-induced emesis: a National Cancer Institute of Canada Clinical Trials Group phase III study. J Clin Oncol 18: 1960-1966, 2000.

4. Herr I and Pfitzenmaier J: Glucocorticoid use in prostate cancer and other solid tumors: implications for effectiveness of cytotoxic treatment and metastases. Lancet Oncol 7: 425-430, 2006.

5. Haid M: Steroid antiemesis may be harmful. N Engl J Med 304: 1237, 1981.

6. Rutz HP: Effects of corticosteroid use on treatment of solid tumours. Lancet 360: 1969-1970, 2002.

7. Iversen $\mathrm{HG}$ and Hjort GH: The influence of corticoid steroids on the frequency of spleen metastases in patients with breast cancer. Acta Pathol Microbiol Scand 44: 205-212, 1958.

8. Sherlock P and Hartmann WH: Adrenal steroids and the pattern of metastases of breast cancer. JAMA 181: 313-317, 1962 
9. Sorensen HT, Mellemkjaer L, Nielsen GL, Baron JA, Olsen JH and Karagas MR: Skin cancers and non-hodgkin lymphoma among users of systemic glucocorticoids: a population-based cohort study. J Natl Cancer Inst 96: 709-711, 2004.

10. Herr I, Ucur E, Herzer K, et al: Glucocorticoid co-treatment induces apoptosis resistance toward cancer therapy in carcinomas. Cancer Res 63: 3112-3120, 2003.

11. Gassler N, Zhang C, Schnabel PA, et al: Dexamethasone-induced cisplatin and gemcitabine resistance in lung carcinoma samples treated ex vivo. Br J Cancer 92: 1084-1088, 2005.

12. Zhang C, Kolb A, Buechler P, et al: Corticosteroid co-treatment induces resistance to chemotherapy in surgical resections, xenografts and established cell lines of pancreatic cancer. BMC Cancer 6: 61, 2006.

13. Zhang C, Kolb A, Mattern J, et al: Dexamethasone desensitizes hepatocellular and colorectal tumours toward cytotoxic therapy. Cancer Lett (in press), 2005.

14. Zhang C, Marme A, Wenger T, et al: Glucocorticoid-mediated inhibition of chemotherapy in ovarian carcinomas. Int J Oncol 2: 551-557, 2006.

15. Zhang C, Mattern J, Haferkamp A, et al: Corticosteroid-induced chemotherapy resistance in urological cancers. Cancer Biol Ther 5: 59-64, 2006

16. Nakamori M, Iwahashi M, Nakamura $M$ and Yamaue $H$ : Clinical benefit of chemosensitivity test for patients with regional lymph node-positive esophageal squamous cell carcinoma. J Surg Oncol 84: 10-16, 2003.
17. Ioannidis JP, Hesketh PJ and Lau J: Contribution of dexamethasone to control of chemotherapy-induced nausea and vomiting a meta-analysis of randomized evidence. J Clin Oncol 18 : 3409-3422, 2000

18. The Italian Group for Antiemetic Research: Dexamethasone, granisetron, or both for the prevention of nausea and vomiting during chemotherapy for cancer. N Engl J Med 332: 1-5, 1995.

19. Brady ME, Sartiano GP, Rosenblum SL, Zaglama NE and Bauguess CT: The pharmacokinetics of single high doses of dexamethasone in cancer patients. Eur J Clin Pharmacol 32: 593-596, 1987.

20. Boumpas DT, Chrousos GP, Wilder RL, Cupps TR and Balow JE: Glucocorticoid therapy for immune-mediated diseases: basic and clinical correlates. Ann Intern Med 119: 1198-1208, 1993.

21. Rasmuson T, Ljungberg B, Grankvist K, Jacobsen J and Olsson T: Increased serum cortisol levels are associated with high tumour grade in patients with renal cell carcinoma. Acta Oncol 40: 83-87, 2001.

22. Fidler IJ, Gersten DM and Kripke ML: Influence of immune status on the metastasis of three murine fibrosarcomas of different immunogenicities. Cancer Res 39: 3816-3821, 1979.

23. Gorelick E, Segal S and Feldman M: Control of lung metastasis progression in mice: role of growth kinetics of 3LL Lewis lung carcinoma and host immune reactivity. J Natl Cancer Inst 65: 1257-1264, 1980.

24. Seshadri M, Poduval TB and Sundaram K: Studies on metastases. I. Role of sensitization and immunosuppression. J Natl Cancer Inst 63: 1205-1209, 1979. 